\title{
MATHEMATICAL MODELING OF CONTACT RESISTANCE IN SILICON PHOTOVOLTAIC CELLS*
}

\author{
J. P. BLACK ${ }^{\dagger}$, C. J. W. BREWARD ${ }^{\dagger}$, P. D. HOWELL ${ }^{\dagger}$, AND R. J. S. YOUNG ${ }^{\ddagger}$
}

\begin{abstract}
In screen-printed silicon-crystalline solar cells, the contact resistance of a thin interfacial glass layer between the silicon and the silver electrode plays a limiting role for electron transport. We analyze a simple model for electron transport across this layer, based on the driftdiffusion equations. We utilize the size of the current/Debye length to conduct asymptotic techniques to simplify the model; we solve the model numerically to find that the effective contact resistance may be a monotonic increasing, monotonic decreasing, or nonmonotonic function of the electron flux, depending on the values of the physical parameters.
\end{abstract}

Key words. drift diffusion, asymptotic analysis, contact resistance, electrochemical systems

AMS subject classifications. 34B15, 34E10, 78A35

DOI. $10.1137 / 130911974$

1. Introduction. The majority of photovoltaic solar panels today involve a silicon emitter which is covered in a silicon nitride passivation layer that also acts as an antireflection coating. During the manufacturing process, silver paste is patterned by screen printing onto the emitter. After printing, the cells are fired in a furnace, where glass added as a binder to the silver paste chemically etches through the silicon nitride through a redox reaction [10]. A schematic of the device is illustrated in Figure 1.1. The presence of these glasses in the paste leads to a thin interfacial glass layer $(10 \mathrm{~nm}-1 \mu \mathrm{m})$ between the bulk silicon and silver electrode that inhibits electron flow $[17,18]$.

To attain low contact resistance between the front side silver electrode and silicon emitter, the emitter must be highly doped with phosphorous atoms at the surface. This is undesirable because a high level of doping leads to an increased amount of electron-hole recombination at the front surface, reducing the efficiency of the solar cell. An enhanced understanding of the local electron transport mechanisms will aid in the development of silver pastes that achieve low contact resistance and, consequently, enable the production of higher efficiency solar cells.

Although it is clear that the glass layer between the silicon emitter and silver electrode plays a limiting role for electron transport across the interface [2], there are two different hypotheses concerning the conduction mechanism of electrons. The first is that electrons flow through silver crystallites embedded in the silicon that are either in direct contact with the silver electrode or separated from it by an ultrathin glass layer $[2,26]$. The second is that silver precipitates present in the interfacial glass layer aid the electron transport across the interface $[8,17]$. Much of the literature to date focuses on the ongoing debate over which of these conduction mechanisms is dominant

*Received by the editors March 5, 2013; accepted for publication (in revised form) July 16, 2013; published electronically October 22, 2013. This work was supported by EPSRC and DuPont (UK) Ltd. through mathematics CASE award BK/10/040. This work was also partially supported by Award KUK-C1-013-04 made by King Abdullah University of Science and Technology (KAUST).

http://www.siam.org/journals/siap/73-5/91197.html

${ }^{\dagger}$ Mathematical Institute, University of Oxford, Oxford, OX2 6GG, UK (black@maths.ox.ac.uk, chris.beward@maths.ox.ac.uk, howell@maths.ox.ac.uk).

${ }^{\ddagger}$ DuPont Microcircuit Materials, DuPont (UK) Limited, Frenchay, Bristol, BS16 1QD, UK (richard.young@dupont.com). 


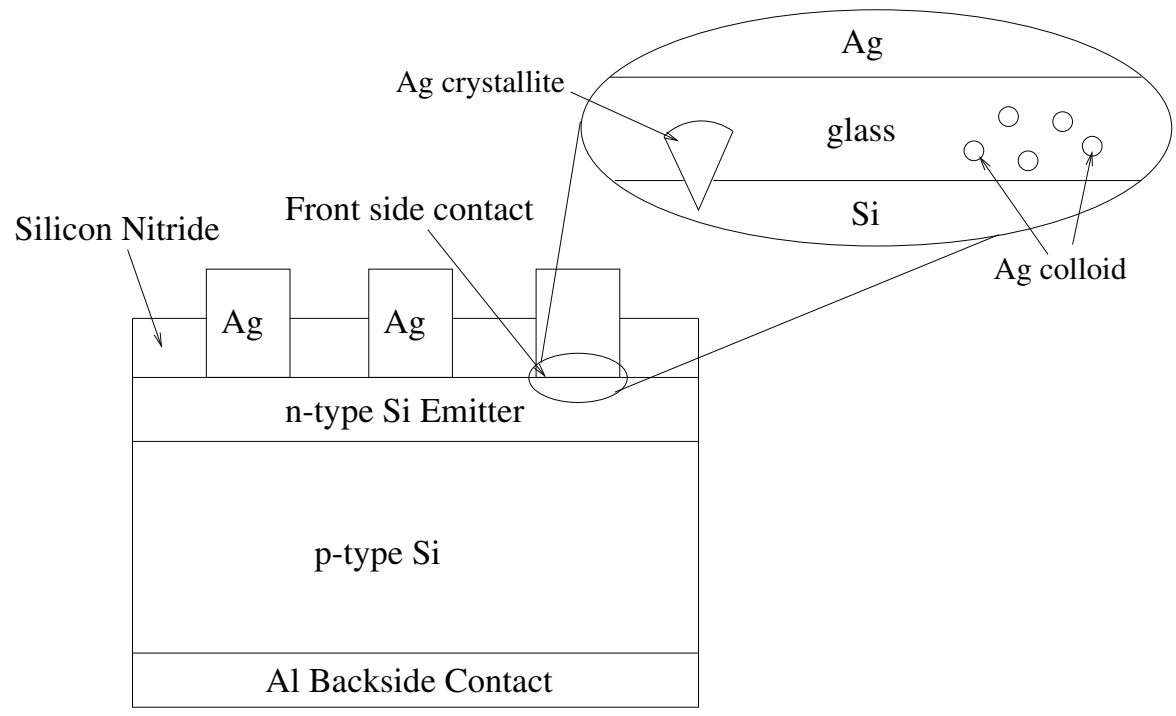

FIG. 1.1. Schematic diagram of a solar cell after firing. We are considering the front side contact between the silver and the n-type silicon emitter.

$[7,8,12,13,15,16,18,19,26]$. The whole glass layer has been reduced to a Schottky barrier by Kim et al. [14], who assume that an increase in the thickness of the glass layer causes the Schottky barrier height to increase. We instead focus our attention on the resistance of the glass layer itself by analyzing a mathematical model of electron flow based on the drift-diffusion equations, while neglecting the complicated boundary effects that occur at the silicon-glass and glass-silver interfaces. This novel approach to modeling contact resistance enables us to determine systematically how the contact resistance changes as the sizes of different parameters are varied.

The drift-diffusion equations are often used to model the current flow in various devices. These can be electrochemical, such as microbatteries or water desalination apparatus, or they can be semiconductor devices, for example, $p$ - $n$ junctions, metaloxide semiconductor (MOS) diodes, transistors, and organic semiconductors; see [3, $5,6,9,20,21,22,24,25]$. In each of these studies, the authors use asymptotic techniques, in particular exploiting the smallness of the Debye length relative to the device length. In this limit it is consistently found that the variations in electric field and charge carrier densities predominantly occur in boundary layers at any interfaces present, except where there is an ohmic contact in the semiconductor devices.

In this paper we formulate a simple mathematical model of electron transport through the glass layer. We first solve the resulting equations analytically and numerically before obtaining a number of approximate expressions for the resistance of the glass layer by using asymptotic techniques. We show that, depending on the values of the physical parameters, the resistance of the glass layer may be a monotonic increasing, monotonic decreasing, or nonmonotonic function of the electron flux.

\section{Mathematical model.}

2.1. Formulation of the problem. We assume that the width of the glass layer is much bigger than its thickness and hence reduce the problem to one-dimensional conduction through a layer of thickness $L$ driven by an imposed electric potential dif- 
ference $\Phi$. We consider two different possible systems, namely a glass layer separating either (i) the silicon emitter from a silver electrode (Si-glass-Ag system), or (ii) a silver crystallite from the silver electrode (Ag-glass-Ag system). In either case, we denote the electron densities at the two interfaces as $n_{0}$ and $n_{1}$. These values will depend on the electron densities and electron affinities in the materials on either side of the glass layer as well as the electron affinity of the glass, and will be affected by the boundary effects at the silicon-glass and glass-silver interfaces but, for the purpose of this study, we take $n_{0}$ and $n_{1}$ to be known constants. In the Ag-glass-Ag system we expect $n_{0} \approx n_{1}$ and in the Si-glass-Ag system we expect $n_{0}<n_{1}$.

2.2. Governing equations. We first assume that the charge is predominantly carried by electrons rather than by holes (for example, in [11] it is found that in $\mathrm{SiO}_{2}$ electron mobility exceeds hole mobility by a factor of $10^{10}$ ). We therefore ignore the motion of holes henceforth. Second, we assume that our system is in a quasi-steady state so that the conservation of electrons leads to the equation

$$
\frac{\mathrm{d} j}{\mathrm{~d} x}=0,
$$

where $j$ is the flux of electrons. The flux $j$ of electrons in the glass layer is assumed to be a combination of drift, driven by the electric field, and diffusion which can be written as

$$
j=-\mu\left(n \frac{\mathrm{d} \phi}{\mathrm{d} x}+\frac{k_{B} T}{q} \frac{\mathrm{d} n}{\mathrm{~d} x}\right),
$$

where $n$ is the density of free electrons, $\phi$ is electric potential, $\mu$ is electron mobility, $k_{B}$ is Boltzmann's constant, $T$ is absolute temperature, and $q$ is the charge on an electron. The electric potential satisfies Poisson's equation

$$
\frac{\mathrm{d}^{2} \phi}{\mathrm{d} x^{2}}=-\frac{q n}{\epsilon}
$$

where $\epsilon$ is the absolute permitivity. The boundary conditions are

$$
n(0)=n_{0}, \quad \phi(0)=\Phi, \quad n(L)=n_{1}, \quad \phi(L)=0 .
$$

Equations (2.2) and (2.3) comprise a third-order system with four boundary conditions (2.4), making the problem overdetermined. In a given situation we can therefore either specify the potential difference $\Phi$ and try to determine the corresponding electron flux $j$ or vice versa.

2.3. Nondimensionalization. The problem is nondimensionalized in the usual way for electrochemical systems, namely,

$$
x=L x^{*}, \quad n=n_{1} n^{*}, \quad \phi=\frac{k_{B} T}{q} \phi^{*}, \quad \Phi=\frac{k_{B} T}{q} \Phi^{*}, \quad j=\frac{\mu k_{B} T n_{1}}{q L} j^{*} .
$$

Substituting (2.5) into (2.2) and (2.3) and dropping the starred notation we obtain

$$
\begin{aligned}
j & =-n \frac{\mathrm{d} \phi}{\mathrm{d} x}-\frac{\mathrm{d} n}{\mathrm{~d} x}, \\
\nu^{2} \frac{\mathrm{d}^{2} \phi}{\mathrm{d} x^{2}} & =-n,
\end{aligned}
$$


where we recall that the electron flux $j$ is constant. The boundary conditions (2.4) become

$$
n(0)=\alpha, \quad \phi(0)=\Phi, \quad n(1)=1, \quad \phi(1)=0 .
$$

The dimensionless parameters are

$$
\alpha=\frac{n_{0}}{n_{1}} \quad \text { and } \quad \nu=\frac{L_{D}}{L}=\sqrt{\frac{\epsilon k_{B} T}{q^{2} L^{2} n_{1}}},
$$

where $L_{D}$ is the Debye length. In the Ag-glass-Ag system we expect $\alpha \approx 1$ and in the Si-glass-Ag system we expect $\alpha<1$. Due to the high electron density present in silver we expect $\nu$ to be small in either case.

2.4. Resistance. Rewriting (2.6) as

$$
j=-n(\phi+\log n)^{\prime}
$$

(here and henceforth using ' as shorthand for $\mathrm{d} / \mathrm{d} x$ ), we see that when there is no electron flow, we can use (2.8) to assert that $\Phi=-\log \alpha$. We therefore define the effective resistance of the layer as

$$
R=\frac{\Phi+\log \alpha}{j}
$$

where we have subtracted the electric potential required to offset the chemical potential $(-\log \alpha)$ from $\Phi$ before dividing by the electron flux. In this paper we take $\Phi>0$ without loss of generality and, motivated by the problem of contact resistance in solar cells, we will only consider cases where $\Phi+\log \alpha>0$. Physically this means that the electrons are always flowing into the silver electrode.

2.5. Analytical and numerical solution. Eliminating $n$ between (2.6) and (2.7), we find

$$
\frac{j}{\nu^{2}}=\phi^{\prime \prime} \phi^{\prime}+\phi^{\prime \prime \prime}
$$

Now integrating (2.12) and using the Riccati substitution (see, for example, [4]) $u=\mathrm{e}^{\phi / 2}$ we find that $u$ satisfies

$$
u^{\prime \prime}(x)=\left(\frac{j x}{2 \nu^{2}}-C\right) u(x),
$$

and the boundary conditions

$$
\begin{array}{ll}
u(0)=\mathrm{e}^{\Phi / 2}, & \alpha=2 \nu^{2}\left(u^{\prime}(0)^{2} \mathrm{e}^{-\Phi}+C\right), \\
u(1)=1, & 1=2 \nu^{2}\left(u^{\prime}(1)^{2}-\frac{j}{2 \nu^{2}}+C\right),
\end{array}
$$

where $C$ is an unknown constant of integration. To derive (2.14) we make use of the relation

$$
n=2 \nu^{2}\left[\left(\frac{u^{\prime}}{u}\right)^{2}-\left(\frac{j x}{2 \nu^{2}}-C\right)\right] .
$$

A similar system is solved in [25]. 

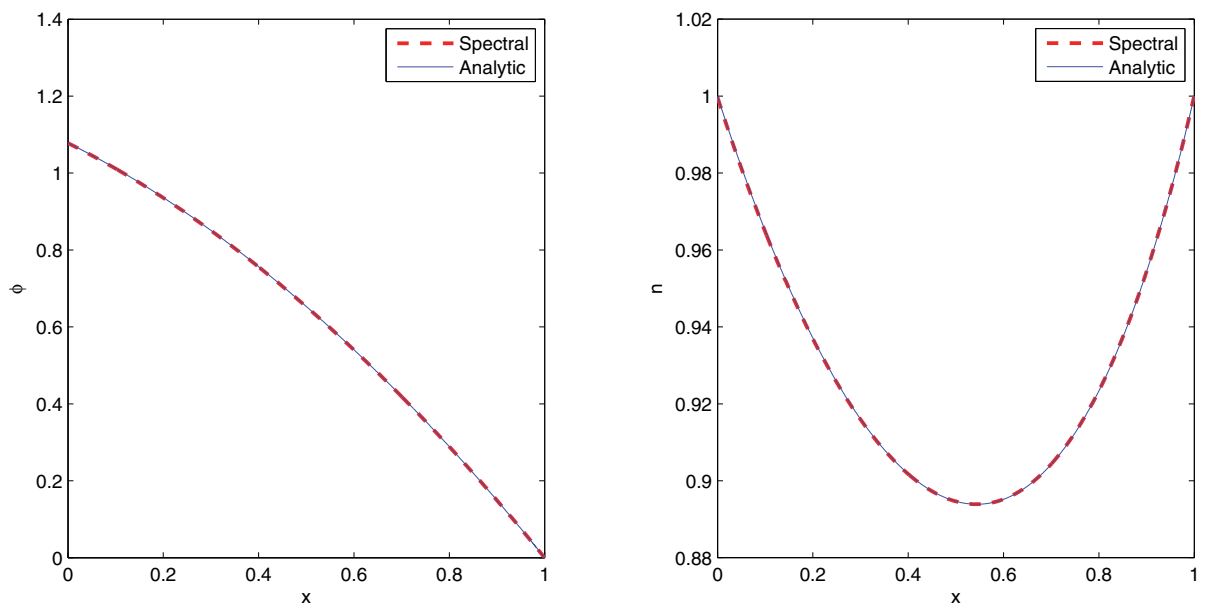

FIG. 2.1. Electric potential $\phi$ and electron density $n$ plotted against position $x$ with parameter values $\nu=1, \alpha=1, j=1$, and $\Phi=1.078$.

The general solution to $(2.13)$ is

$$
u=a \operatorname{Ai}(\chi x-w)+b \operatorname{Bi}(\chi x-w),
$$

where $\mathrm{Ai}$ and $\mathrm{Bi}$ are Airy functions,

$$
w=C\left(\frac{2 \nu^{2}}{j}\right)^{2 / 3}, \quad \chi=\left(\frac{j}{2 \nu^{2}}\right)^{1 / 3},
$$

and $a, b$ are constants of integration, where we note that $j / \nu^{2}$ is a key parameter of the problem. By substituting (2.16) into (2.14), we obtain

$$
\begin{aligned}
a \operatorname{Ai}(\chi-w)+b \operatorname{Bi}(\chi-w) & =1, \\
a \operatorname{Ai}(-w)+b \operatorname{Bi}(-w) & =\mathrm{e}^{\Phi / 2}, \\
2 \chi^{2}\left\{\left(a \operatorname{Ai}^{\prime}(-w)+b \operatorname{Bi}^{\prime}(-w)\right)^{2} \mathrm{e}^{-\Phi}+w\right\} & =\frac{\alpha}{\nu^{2}}, \\
2 \chi^{2}\left\{\left(a \operatorname{Ai}^{\prime}(\chi-w)+b \operatorname{Bi}^{\prime}(\chi-w)\right)^{2}+w-\chi\right\} & =\frac{1}{\nu^{2}} .
\end{aligned}
$$

In principle, elimination of $a, b$, and $w$ between the four transcendental equations (2.18) leads to a functional relation between $j$ and $\Phi$. For example, the numerical solution of (2.18) with $\alpha=1, \nu=1$, and $j=1$ gives $\Phi=1.078$ to three decimal places, and we plot the resulting solutions $\phi(x)$ and $n(x)$ in Figure 2.1. However, this approach is unwieldy in practice: the oscillatory nature of the Airy functions gives rise to multiple solution branches of (2.18), and one must pick the first root to ensure that the solution is physical.

In contrast, it is straightforward to solve the boundary-value problem (2.6)-(2.8) numerically. We rewrite the governing equations (2.6) and (2.7) in the form

$$
\nu^{2} \phi^{\prime \prime}=-n, \quad n^{\prime \prime}=-n^{\prime} \phi^{\prime}+\frac{n^{2}}{\nu^{2}},
$$

Copyright $@$ by SIAM. Unauthorized reproduction of this article is prohibited. 
and use the boundary conditions (2.8), therefore specifying the value of $\Phi$. We then calculate $j$ by rearranging $(2.10)$ to find

$$
j=\frac{\Phi+\log \alpha}{\int_{0}^{1} \frac{\mathrm{d} x}{n(x)}} .
$$

The numerical solution of (2.19) and the evaluation of the integral in (2.20) were both implemented using the spectral software package Chebfun [27, 28]. Figure 2.1 demonstrates the excellent agreement between the analytical and numerical solutions when $\nu=1, \alpha=1, j=1$, and $\Phi=1.078$.

\section{Asymptotic analysis.}

3.1. Overview. In this section, we use asymptotic techniques to find approximate expressions for the resistance of the glass layer as $j$ and $\nu$ vary. Our aim is to determine how the resistance $R$ is affected by the values of the various parameters, where $\nu$ is a material parameter of the system and $j$ will vary as the electric potential changes. Figure 3.1 depicts the different asymptotic expressions for $R$ that will be derived, where the arrows linking different regions show how the regions may be matched by taking appropriate intermediate limits. The presence of "Full Problem" in Figure 3.1 indicates regions where it is not possible to obtain a significantly simplified analytic expression for $R$. For completeness, the expressions for $R$ in the large Debye length limit $(\nu \gg 1)$ are included in Figure 3.1, but we will not spell out their derivation since the mathematics is straightforward and this limit is unlikely to be relevant in practice. In all other limits considered throughout this section we assume $j$ is known and look to determine how $\Phi$ varies.

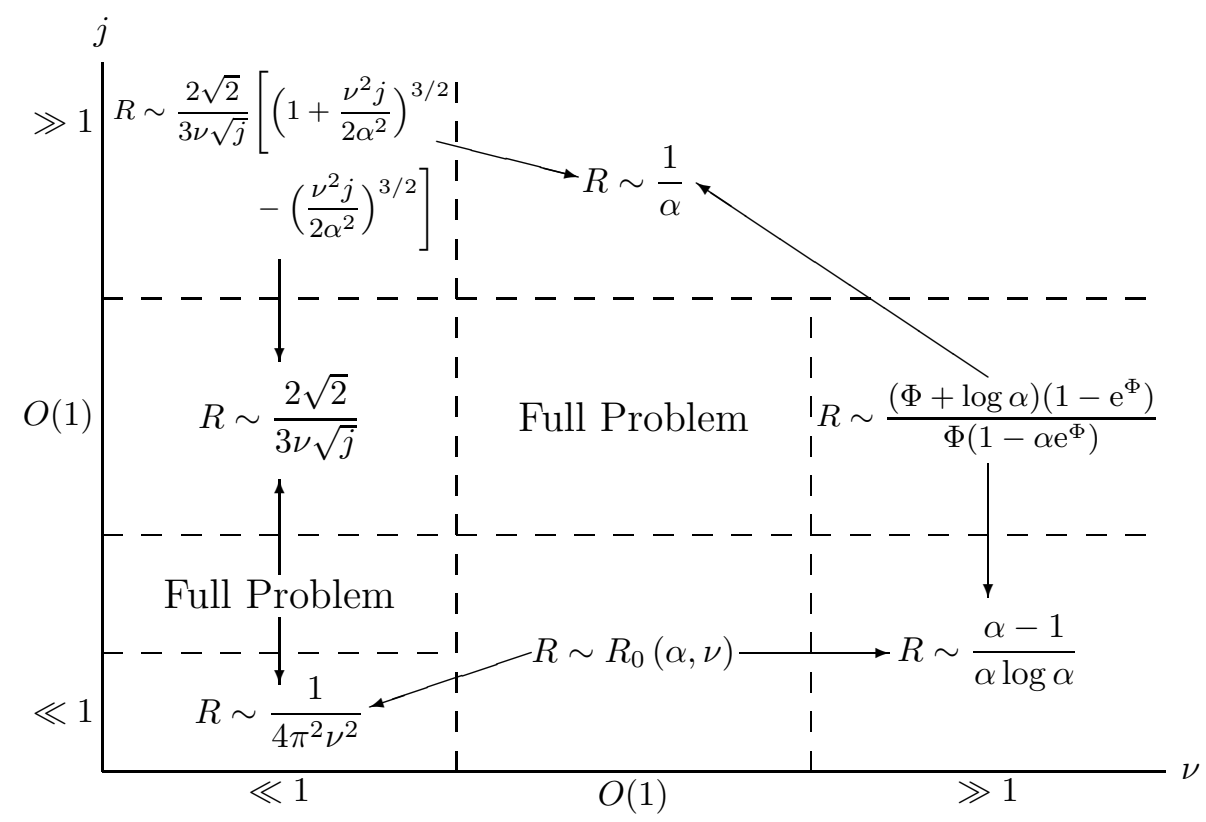

FIG. 3.1. Diagram showing different asymptotic analytic expressions for resistance in different asymptotic regions.

Copyright $@$ by SIAM. Unauthorized reproduction of this article is prohibited. 
3.2. Small electron flux: $j \ll 1$. Here it is helpful to start from the Airy equation (2.13) and boundary conditions (2.14), rewritten in the form

$$
\begin{gathered}
u^{\prime \prime}(x)+C u(x)=\delta x u(x), \\
u(0)=\mathrm{e}^{\Phi / 2}, \quad u^{\prime}(0)^{2}=\left(\frac{\alpha}{2 \nu^{2}}-C\right) \mathrm{e}^{\Phi}, \quad u(1)=1, \quad u^{\prime}(1)^{2}=\frac{1}{2 \nu^{2}}-C+\delta,
\end{gathered}
$$

where $j / 2 \nu^{2}=\delta \ll 1$.

Now we seek the solution $u(x)$ and the constants $\Phi$ and $C$ as asymptotic expansions in powers of $\delta$, that is,

$$
\begin{aligned}
u(x) & \sim u_{0}(x)+\delta u_{1}(x)+\delta^{2} u_{2}(x)+\cdots, \\
\Phi & \sim \Phi_{0}+\delta \Phi_{1}+\delta^{2} \Phi_{2}+\cdots \\
C & \sim C_{0}+\delta C_{1}+\delta^{2} C_{2}+\cdots
\end{aligned}
$$

and therefore

$$
R \sim R_{0}+\delta R_{1}+\cdots
$$

We recall from section 2.4 that

$$
\Phi_{0}=-\log \alpha
$$

is the electric potential at zero electron flux.

To leading order we find

$$
u_{0}=\operatorname{cosec}\left(\sqrt{C_{0}}\right) \sin \left(\sqrt{C_{0}} x\right)+\mathrm{e}^{\Phi_{0} / 2} \operatorname{cosec}\left(\sqrt{C_{0}}\right) \sin \left(\sqrt{C_{0}}(1-x)\right),
$$

and by substituting (3.6) into (3.2) we obtain the relation

$$
\nu^{2}=\frac{\mathrm{e}^{-\Phi_{0} / 2} \sin ^{2}\left(\sqrt{C_{0}}\right)}{4 C_{0}\left[\cosh \left(\Phi_{0} / 2\right)-\cos \left(\sqrt{C_{0}}\right)\right]} .
$$

The integration constant $C_{0}$ is, in principle, determined as a function of $\alpha$ and $\nu$ by (3.5) and the implicit equation (3.7). By requiring $\nu$ to be real and positive, we find that $C_{0}$ lies in the range $\left(-\Phi_{0}^{2} / 4, \pi^{2}\right)$, with $C_{0} \rightarrow-\Phi_{0}^{2} / 4=-(\log \alpha)^{2} / 4$ as $\nu \rightarrow \infty$, and $C_{0} \rightarrow \pi^{2}$ as $\nu \rightarrow 0$.

The limiting resistance (2.11) at zero electron flux is given by

$$
R_{0}=\frac{\Phi_{1}}{2 \nu^{2}}
$$

Fortunately we can avoid the need to calculate the first correction $u_{1}(x)$ by rewriting (3.1) as

$$
\frac{\mathrm{d}}{\mathrm{d} x}\left(u^{\prime 2}+(C-\delta x) u^{2}\right)=-\delta u^{2}
$$

and integrating across the domain to obtain the identity

$$
\mathrm{e}^{\Phi} \alpha-1=2 \delta \nu^{2} \int_{0}^{1} u^{2} \mathrm{~d} x
$$

Copyright $@$ by SIAM. Unauthorized reproduction of this article is prohibited. 


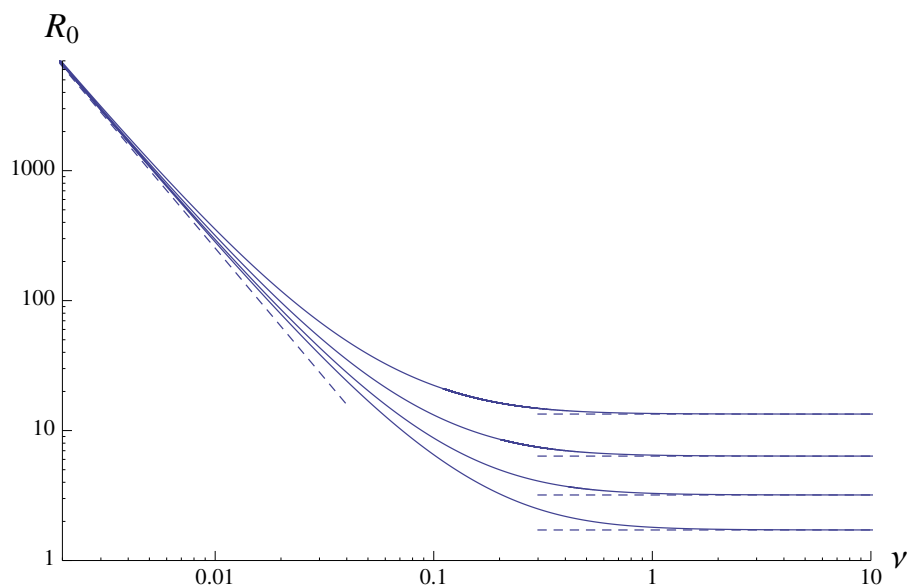

FIG. 3.2. The resistance $R_{0}$ at zero electron flux plotted versus the dimensionless Debye length $\nu$ for different values of the density ratio $\alpha=\mathrm{e}^{-1}, \mathrm{e}^{-2}, \mathrm{e}^{-3}, \mathrm{e}^{-4}$. The dashed curves show the asymptotic limits (3.13) as $\nu \rightarrow 0$ and as $\nu \rightarrow \infty$.

Hence the resistance is given by the exact formula

$$
R=\frac{1}{2 \delta \nu^{2}} \log \left(1+2 \delta \nu^{2} \int_{0}^{1} u(x)^{2} \mathrm{~d} x\right) .
$$

In particular, the limiting resistance as $\delta \rightarrow 0$ may be obtained using only the leadingorder solution:

$$
\begin{aligned}
R_{0} & =\int_{0}^{1} u_{0}(x)^{2} \mathrm{~d} x \\
& =\frac{\mathrm{e}^{\Phi_{0} / 2}\left[\cosh \left(\Phi_{0} / 2\right)-\cos \left(\sqrt{C_{0}}\right)\right]}{\sin ^{2}\left(\sqrt{C_{0}}\right)}-\frac{\mathrm{e}^{\Phi_{0} / 2}\left[\cosh \left(\Phi_{0} / 2\right) \cos \left(\sqrt{C_{0}}\right)-1\right]}{\sqrt{C_{0}} \sin \left(\sqrt{C_{0}}\right)} .
\end{aligned}
$$

For a given value of $\alpha$ (and therefore of $\Phi_{0}$ ), (3.7) and (3.12) determine $R_{0}$ parametrically as a function of $\nu$, with the parameter $C_{0}$ varying in the range $\left(-\Phi_{0}^{2} / 4, \pi^{2}\right)$. This functional relation is plotted in Figure 3.2 for various values of $\alpha$. By analyzing the behavior as $C_{0} \rightarrow \pi^{2}$ and as $C_{0} \rightarrow-\Phi_{0}^{2} / 4$, we obtain the limiting forms

$$
R_{0} \sim \frac{1}{4 \pi^{2} \nu^{2}} \quad \text { as } \nu \rightarrow 0, \quad R_{0} \sim\left\{\begin{array}{ll}
\frac{\alpha-1}{\alpha \log \alpha} & \text { if } \alpha \neq 1 \\
1 & \text { if } \alpha=1
\end{array} \text { as } \nu \rightarrow \infty,\right.
$$

which are plotted as dashed curves in Figure 3.2. The expressions in (3.13) show that, when $\nu \gg 1$, the value of $\alpha$ plays the dominant role in determining the value of $R_{0}$ but, if $\nu$ is decreased sufficiently, the resistance becomes independent of $\alpha$. This implies that the contact resistance will be greater in the Si-glass-Ag system than in the Ag-glass-Ag system, until the glass layer becomes sufficiently thick.

Figure 3.2 demonstrates that the resistance can vary by orders of magnitude depending on the value of $\nu$. In particular, the fact that $R_{0} \rightarrow \infty$ as $\nu \rightarrow 0$ suggests that there may be a new distinguished limit where $j$ and $\nu$ are both small. We will return to this possibility in section 3.4 . 

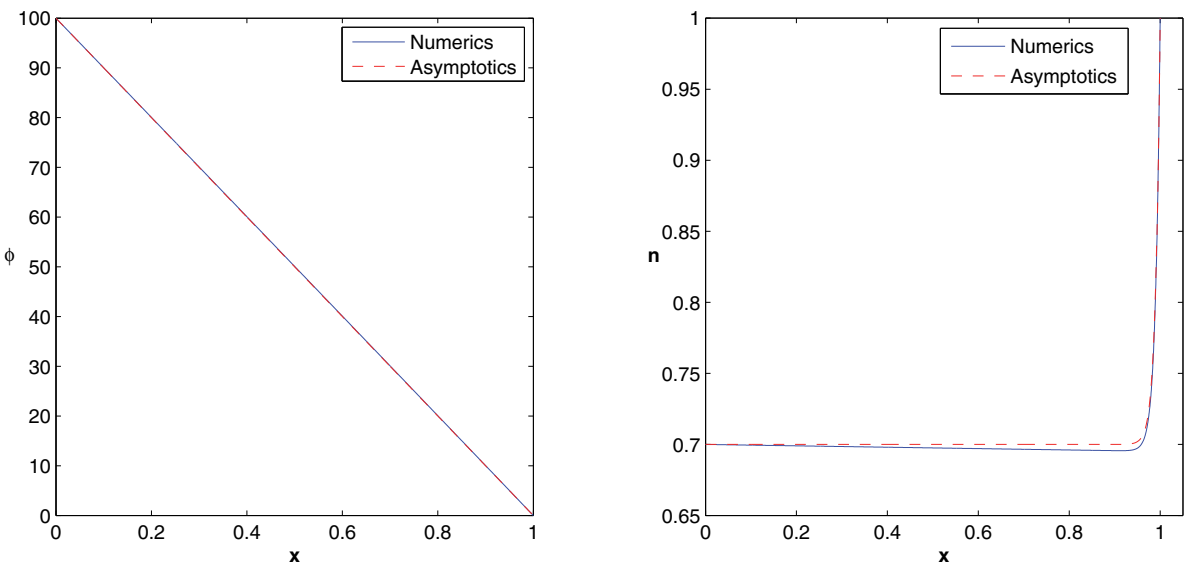

FIG. 3.3. Electric potential $\phi$ and electron density $n$ plotted against position $x$ with parameter values $\nu=1, \alpha=0.7, j=70$, and $\Phi=100$.

3.3. Large electron flux: $j \gg 1$. In the limit of large electron flux $j$, the asymptotic analysis is more straightforward, and one easily obtains the leading-order approximations. We represent $n$ as the composite of the solution found in the boundary layer at $x=1$ and the constant solution found elsewhere in our domain:

$$
\phi \sim \frac{j(1-x)}{\alpha}, \quad n \sim \alpha+(1-\alpha) \mathrm{e}^{-j(1-x) / \alpha} .
$$

Figure 3.3 plots the expressions given in (3.14) and demonstrates their good agreement with the numerical solution. Making use of (2.11), we therefore find that at large $j$ the resistance of the glass layer tends to the constant value

$$
R_{\infty}=\frac{1}{\alpha} .
$$

We conclude that in the situation where the applied potential difference is much greater than the thermal voltage, we will always be in the $j \gg 1$ regime and here the glass behaves like a "normal" resistor (effectively constant contact resistance). We note that neither the leading-order solution (3.14) nor the limiting resistance $R_{0}$ depends on $\nu$. This is due to the high electric potential difference flooding the glass layer with charge carriers so the Debye length is no longer an important factor. Indeed, (3.14) shows that the electron density $n$ is approximately constant outside a boundary layer at $x=1$ whose thickness scales with one over the electric field.

\subsection{Small Debye length: $\nu \ll 1$.}

3.4.1. Introduction. In this section we consider the limit where $\nu \rightarrow 0$, in a similar manner to $[3,5,6,9,20,21,22]$. This corresponds to the situation where the glass layer is much thicker than the Debye length. Examining the governing equations and boundary conditions (2.6)-(2.8), we hypothesize that if $\Phi=O(1)$, in the limit $\nu \rightarrow 0$, then the corresponding electron flux $j=O\left(\nu^{2}\right)$. Also we expect to find that $n=O\left(\nu^{2}\right)$ away from the boundaries and there are boundary layers to satisfy the $O(1)$ boundary conditions for $n$ at $x=0$ and $x=1$. In the following sections we will 
derive and asymptotically match leading-order solutions in the outer region and in each boundary layer.

3.4.2. Boundary layers. First considering the boundary layer at $x=0$, we make the rescaling $x=\nu X_{0}$ before letting $\nu \rightarrow 0$ so that (2.6) and (2.7) become

$$
\begin{aligned}
n \frac{\mathrm{d} \phi}{\mathrm{d} X_{0}}+\frac{\mathrm{d} n}{\mathrm{~d} X_{0}} & =0, \\
\frac{\mathrm{d}^{2} \phi}{\mathrm{d} X_{0}^{2}} & =-n .
\end{aligned}
$$

Substituting (3.17) into (3.16), integrating, and using the substitution $\phi=2 \log \psi$ gives

$$
\frac{\mathrm{d}^{2} \psi}{\mathrm{d} X_{0}^{2}}-\frac{K_{1}}{2} \frac{\mathrm{d} \psi}{\mathrm{d} X_{0}}=0
$$

where $K_{1}$ is a constant of integration. We find $K_{1}=0$ to match with the outer solution, to be discussed later in section 3.4.3, and therefore using the boundary conditions (2.8) we obtain the leading-order solutions

$$
\phi \sim \Phi+2 \log \left(1+X_{0} \sqrt{\frac{\alpha}{2}}\right), \quad n \sim \alpha\left(1+X_{0} \sqrt{\frac{\alpha}{2}}^{-2} .\right.
$$

Considering the boundary layer at $x=1$ we make the substitution $x=1-\nu X_{1}$, and following a similar method will yield the leading-order solution

$$
\phi \sim 2 \log \left(1+\frac{X_{1}}{\sqrt{2}}\right), \quad n \sim\left(1+\frac{X_{1}}{\sqrt{2}}\right)^{-2} .
$$

Since the width of these boundary layers scales with the Debye length $\nu$, they are Debye layers and will be used to provide matching conditions for the outer region analyzed in the following section. The expressions for the boundary layers given in (3.19) and (3.20) along with the outer solution found in section 3.4.3 are shown in Figure 3.4.

3.4.3. Outer solution. Away from the boundary layers, we introduce the variables $N=n / \nu^{2}$ and $J=j / \nu^{2}$, so that (2.6) and (2.7) take the form

$$
J=-N \phi^{\prime}-N^{\prime}, \quad \phi^{\prime \prime}=-N .
$$

We follow a method similar to that outlined in section 2.5; by eliminating $N$ from (3.21) and making the substitution $\phi=2 \log (U / \nu)$, we include the parameter $\nu$ for matching purposes, to obtain the general solution

$$
U(x)=a \operatorname{Ai}(\chi x-w)+b \operatorname{Bi}(\chi x-w),
$$

where $a, b$, and $w$ are integration constants and $\chi=(J / 2)^{1 / 3}=\left(j / 2 \nu^{2}\right)^{1 / 3}$ as in (2.17). This agrees with equation (51) of [9], for example. By matching (3.22) with 

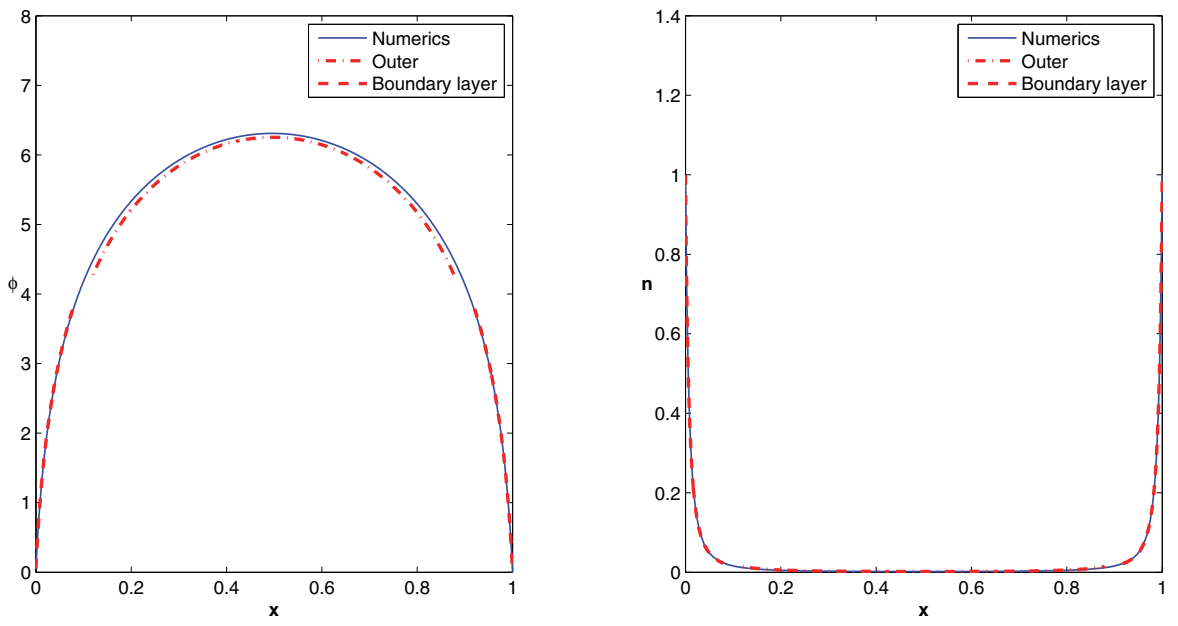

FIG. 3.4. Electric potential $\phi$ and electron density $n$ plotted against position $x$ with parameter values $\nu=0.01, \alpha=1, j=0.0002$, and $\Phi=0.0507$.

(3.19) and (3.20) as $x \rightarrow 0$ and $x \rightarrow 1$, respectively, we obtain the following system of four equations:

$$
\begin{aligned}
a \mathrm{Ai}(-w)+b \operatorname{Bi}(-w) & =0, \\
a \mathrm{Ai}(\chi-w)+b \operatorname{Bi}(\chi-w) & =0, \\
\chi\left\{a \operatorname{Ai}^{\prime}(-w)+b \operatorname{Bi}^{\prime}(-w)\right\} & =\mathrm{e}^{\Phi / 2} \sqrt{\frac{\alpha}{2}}, \\
\chi\left\{a \operatorname{Ai}^{\prime}(\chi-w)+b \operatorname{Bi}^{\prime}(\chi-w)\right\} & =-\frac{1}{\sqrt{2}},
\end{aligned}
$$

which are evidently a simplified limit of the full transcendental system (2.18).

3.4.4. Resistance. Eliminating $a$ and $b$ from (3.23), we get two equations relating $\Phi$ to $\alpha, \chi$, and $w$. It is helpful to combine these into a single complex relation, namely,

$$
-\sqrt{\alpha} \mathrm{e}^{\Phi / 2} \equiv-\mathrm{e}^{j R / 2}=\frac{\operatorname{Ai}(\chi-w)+\mathrm{i} \operatorname{Bi}(\chi-w)}{\operatorname{Ai}(-w)+\mathrm{iBi}(-w)} .
$$

The imaginary part of (3.24) in principle determines $w$ as a function of $\chi$; one must always be careful to choose the smallest (in magnitude) root for $w$ to prevent zeros of $U(x)$ occurring in the interval $[0,1]$ since these would correspond to unphysical singularities in $\phi(x)$. The real part of (3.24) then gives a leading-order approximation for the resistance $R$ in terms of $j$ and $\chi$, which is valid when $j=O\left(\nu^{2}\right)$ and $\nu \ll 1$. In general, therefore, it is still necessary to solve a transcendental equation in this asymptotic regime and there is not a significantly simplified analytic expression for the resistance. However, we can find the small and large electron flux limits by analyzing the relation (3.24) as $\chi \rightarrow 0$ and as $\chi \rightarrow \infty$, respectively.

First taking $\chi \rightarrow 0$, we find that a balance in (3.24) requires $w$ to tend to infinity, specifically $w=O\left(\chi^{-2}\right)$. By expanding (3.24) in powers of $\chi$ and equating the 
imaginary part to zero, we find that

$$
w \sim \frac{1}{\chi^{2}}\left(\pi^{2}+\frac{\chi^{3}}{2}-\frac{\left(15-\pi^{2}\right) \chi^{6}}{48 \pi^{4}}+\frac{\left(1980-210 \pi^{2}+\pi^{4}\right) \chi^{12}}{2304 \pi^{10}}+O\left(\chi^{15}\right)\right)
$$

and

$$
j R \sim \frac{\chi^{3}}{2 \pi^{2}}-\frac{\left(10-\pi^{2}\right) \chi^{9}}{32 \pi^{8}}+O\left(\chi^{15}\right)
$$

as $\chi \rightarrow 0$. Substituting for $\chi$ from (2.17), we thus obtain the approximate resistance in the form

$$
R \sim \frac{1}{4 \pi^{2} \nu^{2}}-\frac{\left(10-\pi^{2}\right) j^{2}}{256 \pi^{8} \nu^{6}}+\cdots,
$$

which is valid when $j \ll \nu^{2} \ll 1$. Hence the resistance again approaches a constant positive value as the electron flux tends to zero, and this reproduces the small- $\nu$ limit of $R_{0}$ found previously in (3.13).

On the other hand, by taking the limit $\chi \rightarrow \infty$ in (3.24), we find that $w \rightarrow-z_{1}$, where $z_{1}$ is the first zero of $\mathrm{Ai}$, and then

$$
\mathrm{e}^{j R / 2} \sim \frac{\operatorname{Bi}\left(\chi+z_{1}\right)}{-\operatorname{Bi}\left(z_{1}\right)}+\text { exponentially small terms. }
$$

Hence we obtain the following approximation for the resistance:

$$
R \sim \frac{2 \sqrt{2}}{3 \nu \sqrt{j}}+O\left(\nu^{-1 / 3} j^{-5 / 6}\right)
$$

which is valid when $\nu \ll 1$ and $j=O(1)$.

The approximation (3.29) suggests that the resistance of the layer falls to zero as the electron flux increases, which is physically unreasonable and contradicts the large electron flux limit (3.15) obtained previously. This implies the existence of a new distinguished limit when $j$ is large and $\nu$ is small, which we examine below.

3.4.5. Distinguished limit: $\nu \ll 1, \nu \sqrt{j}=O(1)$. We recall that the approximation (3.15) was obtained by letting $j \rightarrow \infty$ and then $\nu \rightarrow 0$, while (3.29) was obtained by taking the limits in the reverse order. By comparing the two expressions, we deduce that an intermediate regime will occur when $\nu \rightarrow 0$ with $\nu^{2} j=O(1)$. To analyze this regime, we perform the rescalings

$$
\phi=\nu^{-2} \tilde{\phi}, \quad \Phi=\nu^{-2} \tilde{\Phi}, \quad j=\nu^{-2} \tilde{j},
$$

so that the problem $(2.6)-(2.8)$ becomes

$$
\tilde{j}=-n \frac{\mathrm{d} \tilde{\phi}}{\mathrm{d} x}-\nu^{2} \frac{\mathrm{d} n}{\mathrm{~d} x}, \quad \frac{\mathrm{d}^{2} \tilde{\phi}}{\mathrm{d} x^{2}}=-n
$$

subject to

$$
n(0)=\alpha, \quad \tilde{\phi}(0)=\tilde{\Phi}, \quad n(1)=1, \quad \tilde{\phi}(1)=0 .
$$




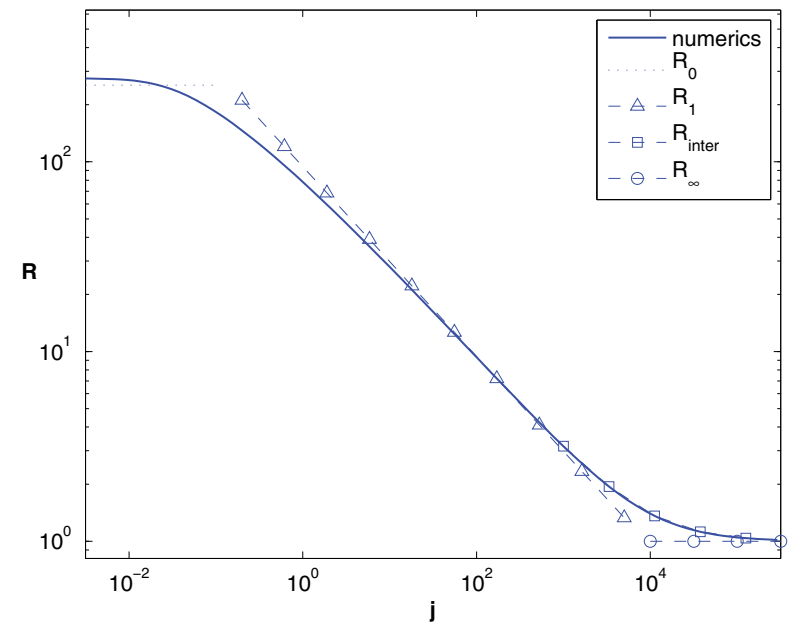

FIG. 3.5. Resistance $R$ plotted against electron flux $j$ with $\alpha=1$ and $\nu=0.01$ : numerical solution (solid line); small- $j$ estimate (3.13) (dotted line); $j \gtrsim 1$ approximation (3.29) (triangles); intermediate approximation (3.34) (squares); large- $j$ limit (3.15) (circles).

The leading-order solutions as $\nu \rightarrow 0$ are

$$
\tilde{\phi}=\frac{2 \sqrt{2 \tilde{j}}}{3}\left[\left(1+\frac{\tilde{j}}{2 \alpha^{2}}\right)^{3 / 2}-\left(x+\frac{\tilde{j}}{2 \alpha^{2}}\right)^{3 / 2}\right] \quad n=\sqrt{\frac{\tilde{j}}{2}}\left(x+\frac{\tilde{j}}{2 \alpha^{2}}\right)^{-1 / 2} .
$$

The boundary condition $n(1)=1$ is not satisfied by (3.33), but this can be resolved by a straightforward boundary layer analysis. Thus, in contrast with the analysis where $j=O\left(\nu^{2}\right)$ carried out above, there is now just one boundary layer at $x=1$. Moreover, as in section 3.3, the boundary layer thickness scales not with the Debye length (i.e., with $\nu$ ) but with one over the electric field.

By evaluating (3.33) at $x=0$ we obtain a leading-order approximation for $\Phi$ in this regime, and hence for the resistance, namely,

$$
R=\frac{2 \sqrt{2}}{3 \nu \sqrt{j}}\left[\left(1+\frac{\nu^{2} j}{2 \alpha^{2}}\right)^{3 / 2}-\left(\frac{\nu^{2} j}{2 \alpha^{2}}\right)^{3 / 2}\right],
$$

valid when $j=O\left(\nu^{-2}\right) \gg 1$. The intermediate approximation (3.34) reduces to (3.29) when $j=O(1)$ and also agrees with (3.15) when $j \nu^{2} \gg 1$.

3.5. Comparison between asymptotics and numerics. In Figure 3.5 we plot the resistance $R$ versus the electron flux $j$ with parameter values $\alpha=1$ and $\nu=0.01$. The solid curve shows the results of numerical solutions obtained using Chebfun. As $j \rightarrow 0$, we see that $R$ approaches a constant value that is well predicted by (3.13), shown as a dotted line. When $1 \ll j \ll \nu^{-2}$, the resistance decreases, approximately obeying the inverse square root law (3.29), plotted using triangles. The transition between these two regimes is governed by the solution of the transcendental equations outlined in section 3.4.4. As $j \rightarrow \infty$, the resistance again approaches a constant value, in agreement with the prediction (3.15), plotted using circles. The transition to this constant value from the power-law behavior is governed by the intermediate asymptotic expression (3.34), plotted using squares. 


\section{Variation of resistance with electron flux.}

4.1. Small- and large-flux limits. In sections 3.2 and 3.3, we showed that the resistance $R$ tends to constant values $R_{0}$ as $j \rightarrow 0$ and as $R_{\infty}$ as $j \rightarrow \infty$. In Figure 3.5 it is evident that $R_{0}>R_{\infty}$ and indeed that $R$ is a monotonic decreasing function of $j$. However, one can easily find values of $\nu$ and $\alpha$ for which the situation is reversed, that is, for which $R$ is an increasing function of $j$ and hence $R_{0}<R_{\infty}$. The curve delineating the regions of $(\alpha, \nu)$ parameter space in which $R_{0}$ exceeds $R_{\infty}$ and vice versa may be found by setting $R_{0}=R_{\infty}$. By combining (3.5), (3.12), and (3.15), we find that the values of $\alpha$ and $C_{0}$ for which $R_{0}=R_{\infty}$ satisfy the equation

$$
\alpha-4 \sqrt{\alpha}\left(\frac{\sqrt{C_{0}} \cos \left(\sqrt{C_{0}}\right)-\sin \left(\sqrt{C_{0}}\right)}{2 \sqrt{C_{0}}-\sin \left(2 \sqrt{C_{0}}\right)}\right)+1-\frac{4 \sqrt{C_{0}} \sin ^{2}\left(\sqrt{C_{0}}\right)}{2 \sqrt{C_{0}}-\sin \left(2 \sqrt{C_{0}}\right)}=0 .
$$

The physical (real positive) root of (4.1) is given by

$$
\alpha=\left(\frac{\sin \left(2 \sqrt{C_{0}}\right)-2 \sqrt{C_{0}} \cos \left(2 \sqrt{C_{0}}\right)}{(2+\sqrt{\Delta}) \sin \left(\sqrt{C_{0}}\right)-2 \sqrt{C_{0}} \cos \left(\sqrt{C_{0}}\right)}\right)^{2},
$$

where $\Delta$ is used as shorthand for the discriminant

$$
\Delta=\left[2 \sqrt{C_{0}}-\sin \left(2 \sqrt{C_{0}}\right)\right]^{2}+4 \sin ^{4}\left(\sqrt{C_{0}}\right) .
$$

Substitution into (3.7) then gives an expression for $\nu^{2}$ when $R_{0}=R_{\infty}$, namely,

$$
\nu^{2}=\frac{\left(\sin \left(2 \sqrt{C_{0}}\right)-2 \sqrt{C_{0}} \cos \left(2 \sqrt{C_{0}}\right)\right)^{2}}{4 C_{0}\left[4 C_{0}+\left(1-\cos \left(2 \sqrt{C_{0}}\right)-2 \sqrt{C_{0}} \sin \left(2 \sqrt{C_{0}}\right)\right)(2+\sqrt{\Delta})\right]} .
$$

Equations (4.2) and (4.4) parameterize the required curve in the $(\alpha, \nu)$-plane; the parameter $C_{0}$ takes values in the range $\left(0, C_{\star}\right)$, where $C_{\star} \approx 5.048$ is the root of the equation $\tan \left(2 \sqrt{C_{\star}}\right)=2 \sqrt{C_{\star}}$ in the range $C \in\left(0, \pi^{2}\right)$. As $C_{0} \rightarrow C_{\star}$, both $\alpha$ and $\nu$ tend to zero, with

$$
\frac{\nu^{2}}{\alpha} \rightarrow \frac{1}{4 C_{\star}}\left(1+\frac{1}{\sqrt{1+4 C_{\star}}}\right) \approx 0.06029 \quad \text { as } \alpha \rightarrow 0 .
$$

On the other hand, as $C_{0} \rightarrow 0$, we find that $\alpha \rightarrow 1$ and $\nu \rightarrow \infty$, with

$$
\nu \sim \frac{1}{\sqrt{6(1-\alpha)}} \quad \text { as } \alpha \rightarrow 1 .
$$

The curve in the $(\alpha, \nu)$-plane parameterized by (4.2) and (4.4) is shown as a solid curve in Figure 4.1. For parameter values lying above this curve, $R_{0}<R_{\infty}$ so the effective resistance of the layer increases as the electron flux increases. However, for values lying below the curve, we will have $R_{0}>R_{\infty}$. In particular, this will inevitably occur if we let $\nu \rightarrow 0$ with $\alpha$ held fixed, in agreement with the analysis of section 3.4. Finally, for specially chosen parameter values lying on the solid curve, the limiting resistances at small and large values of $j$ should be equal. This prediction is validated in Figure 4.2, where, having chosen parameter values that satisfy (4.2) and (4.4), we find that the numerical solution gives the result $R_{0}=R_{\infty}$. 


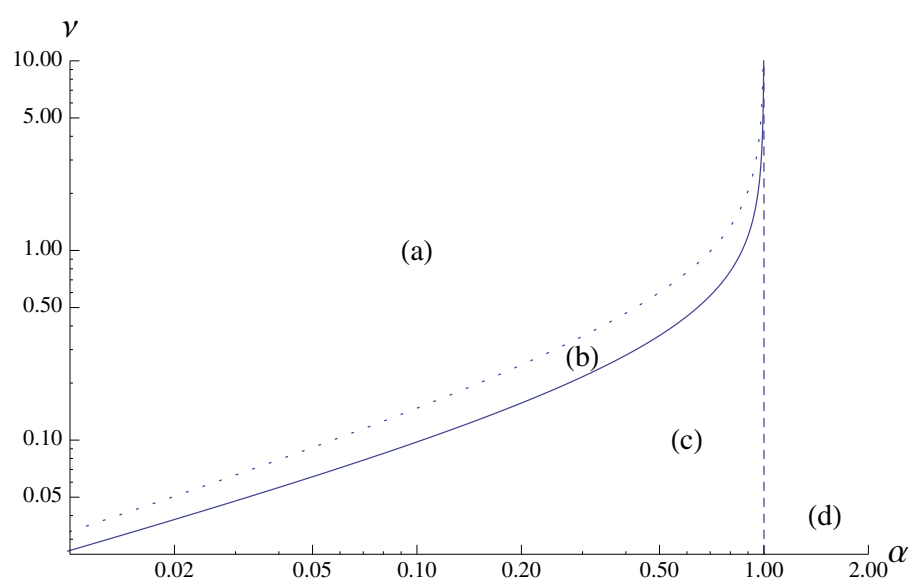

Fig. 4.1. Parameter space of dimensionless Debye length $\nu$ versus electron density ratio $\alpha$. The solid curve, given parametrically by (4.2) and (4.4) shows the parameter values for which $R_{0}=$ $R_{\infty}$. The dashed line indicates $\alpha=1$, for which the first-order correction $R_{1}$ to the resistance as $j \rightarrow 0$ is zero. The dotted curve plots the values satisfying $\nu=(-\alpha / 2 \log \alpha)^{1 / 2}$, for which the first-order correction to the large- $j$ expansion (4.19) is zero. The labels (a)-(d) indicate the regions of parameter space in which the qualitative behavior of the resistance versus electron flux resembles the corresponding plots in Figure 4.3.

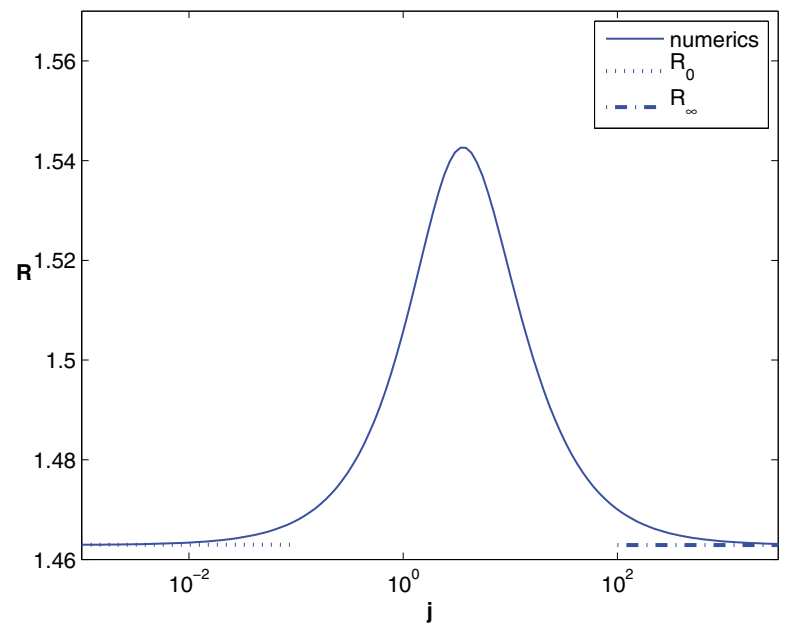

FIG. 4.2. Resistance $R$ plotted against electron flux $j$ with parameter values $\alpha=0.683578$ and $\nu=0.55342$.

\subsection{Monotonicity of the resistance function.}

4.2.1. Introduction. In Figure 3.5 we illustrated that, when $\nu$ is small, the resistance $R$ is typically a monotonic decreasing function of the electron flux $j$. However, the analysis of section 4.1 shows that, for larger values of $\nu$, the large electron flux resistance $R_{\infty}$ may exceed the small electron flux limit $R_{0}$, so that the trend may be reversed. Figure 4.2 demonstrates the even more interesting and unexpected possibility that $R$ may be a nonmonotonic function of $j$ at intermediate values of $\nu$.

Copyright (C) by SIAM. Unauthorized reproduction of this article is prohibited. 
In sections 3.2 and 3.3, we found the constant values approached by $R$ as $j \rightarrow 0$ and as $j \rightarrow \infty$, respectively. Below we will revisit these asymptotic analyses to determine the first-order variations in $j$ and hence the direction in which $R$ approaches each of its limiting values. This will enable us to ascertain the generic forms that the resistance-versus-flux curve may take, and which regions of $(\alpha, \nu)$ parameter space correspond to each possible form.

4.2.2. Small electron flux: $j \ll 1$. Continuing the small- $j$ expansion from section 3.2 to first order, we use (3.11) and (3.4) to obtain the relation

$$
R_{1}=-\nu^{2} R_{0}^{2}+2 \int_{0}^{1} u_{0}(x) u_{1}(x) \mathrm{d} x,
$$

and we recall that $\delta=j / 2 \nu^{2} \ll 1$. Our aim here is to determine the parameter values that give $R_{1}=0$ and therefore delineate the regions of parameter space in which $R(j)$ is an increasing or a decreasing function as $j \rightarrow 0$.

It transpires that the transition where $R_{1}$ goes through 0 is at $\alpha=1$; we therefore proceed assuming $\alpha=1$ (i.e., $\Phi=0$ ) and verify that $R_{1}=0$, so that

$$
u_{0}(x)=\frac{\cos \left(\sqrt{C_{0}}(x-1 / 2)\right)}{\cos \left(\sqrt{C_{0}} / 2\right)} .
$$

In particular, we note for future reference the reflectional symmetry of $u_{0}(x)$ about $x=1 / 2$.

The governing equation for the first-order correction $u_{1}(x)$ is

$$
u_{1}^{\prime \prime}+C_{0} u_{1}=\left(x-C_{1}\right) u_{0},
$$

with boundary conditions

$$
\begin{array}{ll}
u_{1}(0)=\nu^{2} R_{0}, & 2 u_{0}^{\prime}(0) u_{1}^{\prime}(0)=\left(1-2 \nu^{2} C_{0}\right) R_{0}-C_{1}, \\
u_{1}(1)=0, & 2 u_{0}^{\prime}(1) u_{1}^{\prime}(1)=1-C_{1}
\end{array}
$$

when $\alpha=1$. By multiplying (4.9) by $u_{0}(x)$ and integrating over the domain, where we make use of $u_{0}^{\prime \prime}=-C_{0} u_{0}$, we find

$$
\int_{0}^{1}\left(x-C_{1}\right) u_{0}^{2} \mathrm{~d} x=\left[u_{0} u_{1}^{\prime}-u_{0}^{\prime} u_{1}\right]_{0}^{1} .
$$

Now (3.7) and (4.8) and boundary conditions (4.10) enable us to obtain the relation

$$
\int_{0}^{1}\left(x-C_{1}\right) u_{0}^{2} \mathrm{~d} x=\frac{\left(2 C_{1}-1\right)}{2 \sqrt{C_{0}}} \cot \left(\sqrt{C_{0}} / 2\right),
$$

and then by exploiting the symmetry of $u_{0}(x)$ pointed out above, we deduce that $C_{1}=1 / 2$. Hence the general solution of (4.9) can be written in the form

$$
u_{1}(x)=P(x)+A u_{0}(x)+B u_{0}^{\prime}(x),
$$

where $A$ and $B$ are integration constants and $P(x)$ is a particular solution to (4.9), which may be chosen to be antisymmetric about $x=1 / 2$. By evaluating $u_{1}(x)$ at $x=0$ and $x=1$ and again using symmetry arguments, we deduce that

$$
\frac{u_{1}(0)+u_{1}(1)}{2}=A=\frac{\nu^{2} R_{0}}{2} .
$$

Copyright (c) by SIAM. Unauthorized reproduction of this article is prohibited. 


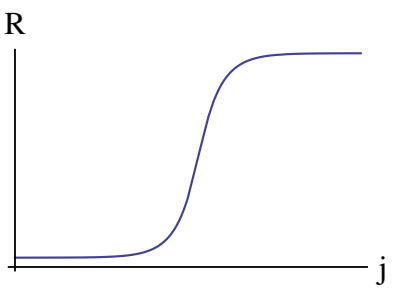

(a)

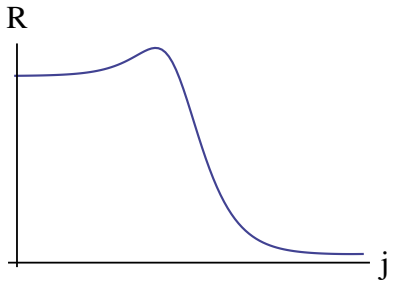

(c)

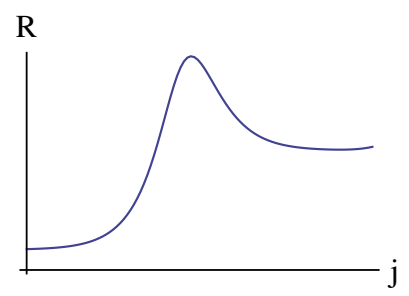

(b)

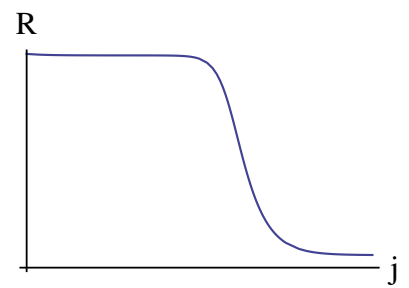

(d)

FIG. 4.3. Schematic showing the four possible qualitative behaviors of the resistance $R$ as a function of the electron flux $j$. The legends (a)-(d) refer to the regions of the $(\alpha, \nu)$ parameter labeled in Figure 4.1 in which each behavior occurs: (a) $R$ is a monotonic increasing function of $j$; (b) $R$ is a nonmonotonic function of $j$ and $R_{\infty}>R_{0}$; (c) $R$ is a nonmonotonic function of $j$ and $R_{0}>R_{\infty}$; (d) $R$ is a monotonic decreasing function of $j$.

Finally, multiplying (4.13) by $u_{0}$, integrating across the domain, and again noting the symmetries of the terms in the integrand, we find that

$$
2 \int_{0}^{1} u_{0}(x) u_{1}(x) \mathrm{d} x=2 A \int_{0}^{1} u_{0}(x)^{2} \mathrm{~d} x=\nu^{2} R_{0}^{2} .
$$

Substitution into (4.7) reveals that $R_{1}=0$ (in this case when $\alpha=1$ ). We infer that the resistance is an increasing function of $j$ when $j \ll 1$ provided $\alpha<1$, as in Figure 4.2. However, if $\alpha>1$, then $R$ will be a decreasing function of $j$ when $j \ll 1$.

4.2.3. Large electron flux: $j \gg 1$. At the opposite limit of large electron flux, we continue the expansions started in section 3.3 to obtain the approximations

$\phi \sim \frac{j}{\alpha}(1-x)+\frac{\alpha}{2 \nu^{2}}\left(1-x^{2}\right)+\cdots$,

$n \sim \alpha+(1-\alpha) \mathrm{e}^{-j(1-x) / \alpha}-\frac{\alpha}{\nu^{2}}(1-\alpha)(1-x) \mathrm{e}^{-j(1-x) / \alpha}+\frac{\alpha^{3}}{\nu^{2} j}\left(e^{-j(1-x) / \alpha}-x\right)+\cdots$

as $j \rightarrow \infty$. The corresponding electric potential is given by

$$
\Phi \sim \frac{j}{\alpha}+\frac{\alpha}{2 \nu^{2}}+\cdots,
$$

and hence the resistance may be expanded in the form

$$
R \sim \frac{1}{\alpha}+\frac{1}{j}\left(\log \alpha+\frac{\alpha}{2 \nu^{2}}\right)+\cdots \quad \text { as } j \rightarrow \infty .
$$

Copyright (c) by SIAM. Unauthorized reproduction of this article is prohibited. 
The direction in which $R$ tends to its limiting value $1 / \alpha$ is determined by the coefficient of $1 / j$ in this expansion. If $\alpha>1$, then this coefficient is necessarily positive and $R$ must therefore approach $1 / \alpha$ from above. The same qualitative behavior will occur if $\alpha<1$ and $\nu^{2}<-\alpha / 2 \log \alpha$. However, if $\alpha<1$ and $\nu^{2}>-\alpha / 2 \log \alpha$, then the resistance increases towards $1 / \alpha$ as $j \rightarrow \infty$.

4.2.4. Summary. Our analysis reveals that the behavior of the resistance $R$ as a function of the electron flux $j$ can follow one of four possible behaviors, illustrated schematically in Figure 4.3. The region of $(\alpha, \nu)$ parameter space where each behavior occurs is labeled (a)-(d) in Figure 4.1. In (a) the resistance increases monotonically with $j$, while in (d) it decreases monotonically. Between these two extremes, the behavior is nonmonotonic, with $R$ decreasing as $j \rightarrow 0$ and as $j \rightarrow \infty$ and, therefore, achieving its maximum value at an intermediate value of $j$. In this case, the limiting values $R_{0}$ as $j \rightarrow 0$ and $R_{\infty}$ as $j \rightarrow \infty$ may satisfy either $R_{0}<R_{\infty}$ (b) or $R_{0}>R_{\infty}$ (c).

5. Conclusion and discussion. In this paper we analyzed a mathematical model for steady electron transport through a glass layer residing between two electrodes. Our key modeling assumptions are that the charge is predominantly carried by electrons, the electron flow is one-dimensional and governed by drift and diffusion, and the electron densities on either side of the glass layer are known. For given values of the physical parameters, the model determines either the electron flux as a function of the applied potential difference or vice versa. The effective resistance $R$ of the layer is then defined as the ratio of the jump in total potential (combining the electric and chemical potentials) to the corresponding electron flux $j$.

Via detailed asymptotic analysis, we show that $R$ approaches constant values as $j$ tends to zero and to infinity and, therefore, behaves like a normal resistor in these regimes. In the limit of small electron flux, the electron density and potential vary consistently across the layer and the limiting resistance $R_{0}$ is related to the parameters $\alpha$ and $\nu$ by (3.7) and (3.12). However, for large electron flux, the charge is approximately uniform across the layer except in a boundary layer at the downstream interface, where it varies rapidly. The potential in this limit varies linearly with distance, and the layer acts like a simple resistor with limiting resistance $R_{\infty}=1 / \alpha$.

We also analyze the limit $\nu \rightarrow 0$, corresponding to the Debye length being much smaller than a typical device length-scale, as is likely to be the case in practice. In this regime, we find that the charge is small outside Debye layers which exist at both interfaces. This layered type solution is often found in the literature, although other authors, for example [9, 24, 25], sometimes find a multilayered solution which does not manifest itself in any of the parameter regimes we have explored. Although there is not, in general, a simple explicit formula for the resistance in this case, we identify successive asymptotic regions in which $R$ is approximately constant for small $j$, decreases like $j^{-1 / 2}$ for intermediate values, then finally approaches a different constant value as $j \rightarrow \infty$. The limiting values for small and large electron flux are consistent with the values $R_{0}$ and $R_{\infty}$ found previously.

When $\nu$ is small, we find that $R_{0}$ exceeds $R_{\infty}$ by a large factor of order $\nu^{-2}$. However, for larger values of $\nu$, it is possible for $R_{0}$ to be equal to or less than $R_{\infty}$. For general values of the parameters $\nu$ and $\alpha$, we find that $R$ may increase or decrease as a function of $j$ or, more surprisingly, achieve a maximum value at some intermediate value of $j$. The four possible generic behaviors of the function $R(j)$ are shown schematically in Figure 4.3, and the regions of $(\alpha, \nu)$ parameter space where each occurs are shown in Figure 4.1. 
In this paper we have discovered that the resistance of a poorly conducting material, when situated between two relatively good conductors, exhibits many interesting phenomena. Our work lays the foundation for accurately modeling the contact mechanism in silicon photovoltaic cells. However, our simple model is based on a number of assumptions that should be revisited to make it more realistic in practice. The neglect of holes in the glass needs to be considered more carefully when the Debye length is small, since strong electric forces may pull the holes into the Debye layer to neutralize the free electrons. Also we should reformulate our idealized Dirichlet boundary conditions to take into account the Schottky barrier type effects that are likely to occur at the interface between two materials with differing Fermi levels, as in [21]. This is of interest since Chu and Bazant [9] found, in their analogous work on electrochemical cells, that the type of boundary conditions may have a large effect on the solution structure. Furthermore, the thickness of the glass layer can be as thin as $10 \mathrm{~nm}$ and at this length-scale it is possible that quantum tunneling effects will start to play a significant role. Such effects may be modeled using a modified drift-diffusion theory with an additional longer-range interaction term incorporated in (2.2); see, for example, $[1,23]$.

\section{REFERENCES}

[1] M. G. Ancona And H. F. Tiersten, Macroscopic physics of the silicon inversion layer, Phys. Rev. B (3), 35 (1987), pp. 7959-7965.

[2] C. Ballif, D. M. Huljic, G. Willeke, and A. Hessler-Wyser, Silver thick-film contacts on highly doped n-type silicon emitters: Structural and electronic properties of the interface, Applied Physics Letters, 82 (2003), 1878.

[3] M. Z. BazAnt, K.T. Chu, AND B. J. BAyly, Current-voltage relations for electrochemical thin films, SIAM J. Appl. Math., 65 (2005), pp. 1463-1484.

[4] C. M. Bender And S. A. Orszag, Advanced Mathematical Methods for Scientists and Engineers, Springer-Verlag, New York, 1999.

[5] P. M. Biesheuvel and M. Z. Bazant, Nonlinear dynamics of capacitive charging and desalination by porous electrodes, Phys. Rev. E (3), 81 (2010), 031502.

[6] A. Bonnefont, F. Argoul, and M. Z. Bazant, Analysis of diffuse-layer effects on timedependent interfacial kinetics, Journal of Electroanalytical Chemistry, 500 (2001), pp. 5261 .

[7] E. Cabrera, S. Olibet, J. Glatz-Reichenbach, R. Kopecek, D. Reinke, and G. Schubert, Current transport in thick film Ag metallization: Direct contacts at silicon pyramid tips?, Energy Procedia, 8 (2011), pp. 540-545.

[8] L. K. Cheng, L. Liang, and Z. Li, Nano-Ag colloids tunneling mechanism for current conduction in front contact of crystalline Si solar cells, in Proceedings of the 34th IEEE Photovoltaics Specialists Conference (PVSEC), Philadelphia, PA, 2009, pp. 2344-2348.

[9] K. T. Chu And M. Z. Bazant, Electrochemical thin films at and above the classical limiting current, SIAM J. Appl. Math., 65 (2005), pp. 1485-1505.

[10] K. Hong, S. Cho, J. You, J. Jeong, S. Bea, and J. Huh, Mechanism for the formation of Ag crystallites in the Ag thick-film contacts of crystalline Si solar cells, Solar Energy Materials and Solar Cells, 93 (2009), pp. 898-904.

[11] R. C. Hughes, Hole mobility and transport in thin SiO2 films, Applied Physics Letters, 26 (1975), pp. 436-438.

[12] A. S. Ionkin, B. M. Fish, Z. R. Li, M. Lewittes, P. D. Soper, J. G. Pepin, And A. F. CARroll, Screen-printed silver pastes with metallic nano-zinc and nano-zinc alloys for crystalline silicon photovoltaic cells, Applied Materials \& Interfaces, 3 (2011), pp. 606611.

[13] M.-I. Jeong, S.-E. Park, D.-H. Kim, J.-S. Lee, Y.-C. Park, K.-S. Ahn, And C.-J. Choi, Transmission electron microscope study of screen-printed Ag contacts on crystalline Si solar cells, Journal of the Electrochemical Society, 157 (2010), pp. H934-H936.

[14] S. J. Kim, S. Y. Kim, J. M. Park, J. N. Heo, J. H. Lee, S. M. Lee, D. H. Kim, W. T. Kim, K. R. Lim, D. Kim, S. C. Park, H. K. Kim, M. C. Song, J. Park, S. S. Jee, and E.-S. LEE, Exploiting metallic glasses for $19.6 \%$ efficient back contact solar cell, Applied 
Physics Letters, 101 (2012), 064106.

[15] S. Kontermann, R. Preu, and G. Willeke, Calculating the specific contact resistance from the nanostructure at the interface of silver thick film contacts on n-type silicon, Applied Physics Letters, 99 (2011), 111905.

[16] S. Kontermann, G. Willeke, and J. Bauer, Electronic properties of nanoscale silver crystals at the interface of silver thick film contacts on n-type silicon, Applied Physics Letters, 97 (2010), 191910.

[17] Z. G. Li, L. Liang, And L. K. Cheng, Electron microscopy study of front-side Ag contact in crystalline Si solar cells, Journal of Applied Physics, 105 (2009), 066102.

[18] Z. G. Li, L. Liang, A. S. Ionkin, B. M. Fish, M. E. Lewittes, L. K. Cheng, and K. R. Mikeska, Microstructural comparison of silicon solar cells' front-side Ag contact and the evolution of current conduction mechanisms, Journal of Applied Physics, 110 (2011), 074304 .

[19] C.-H. Lin, S.-Y. Tsai, S.-P. Hsu, And M.-H. HsieH, Investigation of Ag-bulk/glassy-phase/Si heterostructures of printed Ag contacts on crystalline Si solar cells, Solar Energy Materials and Solar Cells, 92 (2008), pp. 1011-1015.

[20] P. A. Markowich, A singular perturbation analysis of the fundamental semiconductor device equations, SIAM J. Appl. Math., 44 (1984), pp. 896-928.

[21] P. A. Markowich, C. A. Ringhofer, and C. Schmeiser, An asymptotic analysis of onedimensional models of semiconductor devices, IMA J. Appl. Math., 37 (1986), pp. 1-24.

[22] P. A. Markowich AND C. SchmeIser, Uniform asymptotic representation of solutions of the basic semiconductor-device equations, IMA J. Appl. Math., 36 (1986), pp. 43-57.

[23] R. Pinnau, A review on the quantum drift diffusion model, Transport Theory Statist. Phys., 31 (2002), pp. 367-395.

[24] C. P. Please, An analysis of semiconductor P-N junctions, IMA J. Appl. Math., 28 (1982), pp. 301-318.

[25] G. Richardson, C. P. Please, J. Foster, and J. Kirkpatrick, Asymptotic solution of a model for bilayer organic diodes and solar cells, SIAM J. Appl. Math., 72 (2012), pp. 17921817.

[26] G. Schubert, Thick Film Metallization of Crystalline Silicon Solar Cells, Ph.D. thesis, University of Konstanz, Konstanz, Germany, 2006.

[27] L. N. Trefethen, Approximation Theory and Approximation Practice, SIAM, Philadelphia, 2013.

[28] L. N. Trefethen et Al., Chebfun Version 4.2, The Chebfun Development Team, 2011, available online at http://www.maths.ox.ac.uk/chebfun/.

Copyright (c) by SIAM. Unauthorized reproduction of this article is prohibited. 